\title{
Abscess of cauda equina presenting as lumboischialgic pain: a case report
}

\author{
Tomaz Velnar, Gorazd Bunc \\ Department of Neurosurgery, University Medical Centre Maribor, Slovenia
}

\begin{abstract}
Spinal intradural abscess is an uncommon form of pathology carrying poor prognosis, unless treated immediately. We report a case of a patient treated for intense lower back pain and neurological symptoms of foot paresis simulating a herniated lumbar disc. It was evident after image diagnostic procedure and surgery that an intradural abscess of cauda equina was the cause.
\end{abstract}

Key words: cauda equina, intradural abscess, surgery, lumbar pain.

\section{Introduction}

In clinical practice, lumboischialgic pain is a frequently encountered condition that may be caused by numerous factors [9]. It represents an important cause of morbidity and mortality and is most commonly connected to the degenerative processes of the intervertebral disc, although a variety of other rare causes have been described [2,5,9]. Infrequently, spinal abscess may be the cause of sciatica and may demonstrate similar symptoms, thus being easily mistaken for another form of pathology [5]. Initial diagnosis may include spinal degenerative conditions, musculoskeletal injuries, malignancy and infectious agents, most often discitis or vertebral osteomyelitis as well as some of the rarer medical emergencies $[2,3,5]$.

Spinal intradural abscess is an uncommon clinical entity with poor prognosis, unless treated immediately $[4,11]$. Only a few cases have been described, mostly in connection with tuberculosis. The diagnosis and treatment are not easy to perform and when not accomplished early, the consequences may be devastating, leading to a neurological impairment $[1,6,10$, 19]. We report a case of a young and previously healthy patient presenting to the emergency department due to intense lower back pain and neurological symptoms of foot paresis that were caused by an intradural abscess located in cauda equina.

\section{Case report}

A 32-year-old man was admitted to the emergency department due to lumboischialgic pain, which he had been experiencing for approximately five months. The pain was radiating down the anterior and lateral aspect of the left leg to the foot and was progressing in the intensity in the last few weeks before admission. The patient complained of walking difficulties and 
lowered muscle strength in the left foot and of tiredness and apathy that was intensifying in the last weeks. Neither sensory complaints nor bowel and bladder disorders were reported by the patient. No elevated body temperature or chills were experienced. He was healthy previously, suffering only from chronic headache. For this reason, a lumbar puncture was performed six months before and the cerebrospinal fluid laboratory result was normal.

Neurological examination found a decreased strength and limited range of dorsal flexion of the left foot and toe. The strength of all other muscle groups on lower limbs was normal and all the reflexes were symmetrical. The Lassegue test was negative, no sensory disturbances were found and the sphincter function was good. In the general examination, the man was afebrile and looked healthy. Additionally, all the laboratory findings were within the normal range, including the values of white cell count, C-reactive protein and sedimentation rate. The working diagnosis after the clinical and laboratory examination was the herniated lumbar disc at L5-S1 level.

On the contrary, on MRI imaging, an intradural tumourous formation of $2 \mathrm{~cm}$ in diameter was seen in the region of cauda equina at the level of the second lumbar vertebra, slightly on the left side of the spinal cord, compressing the neural structures (Figs. 1, 2). No disc pathology was found. The intradural formation was radiologically classified as intradural neurinoma.

An operation was indicated. A classical laminectomy at the level of the second lumbar vertebra was performed. The dura was cut longitudinally. An elastic oval
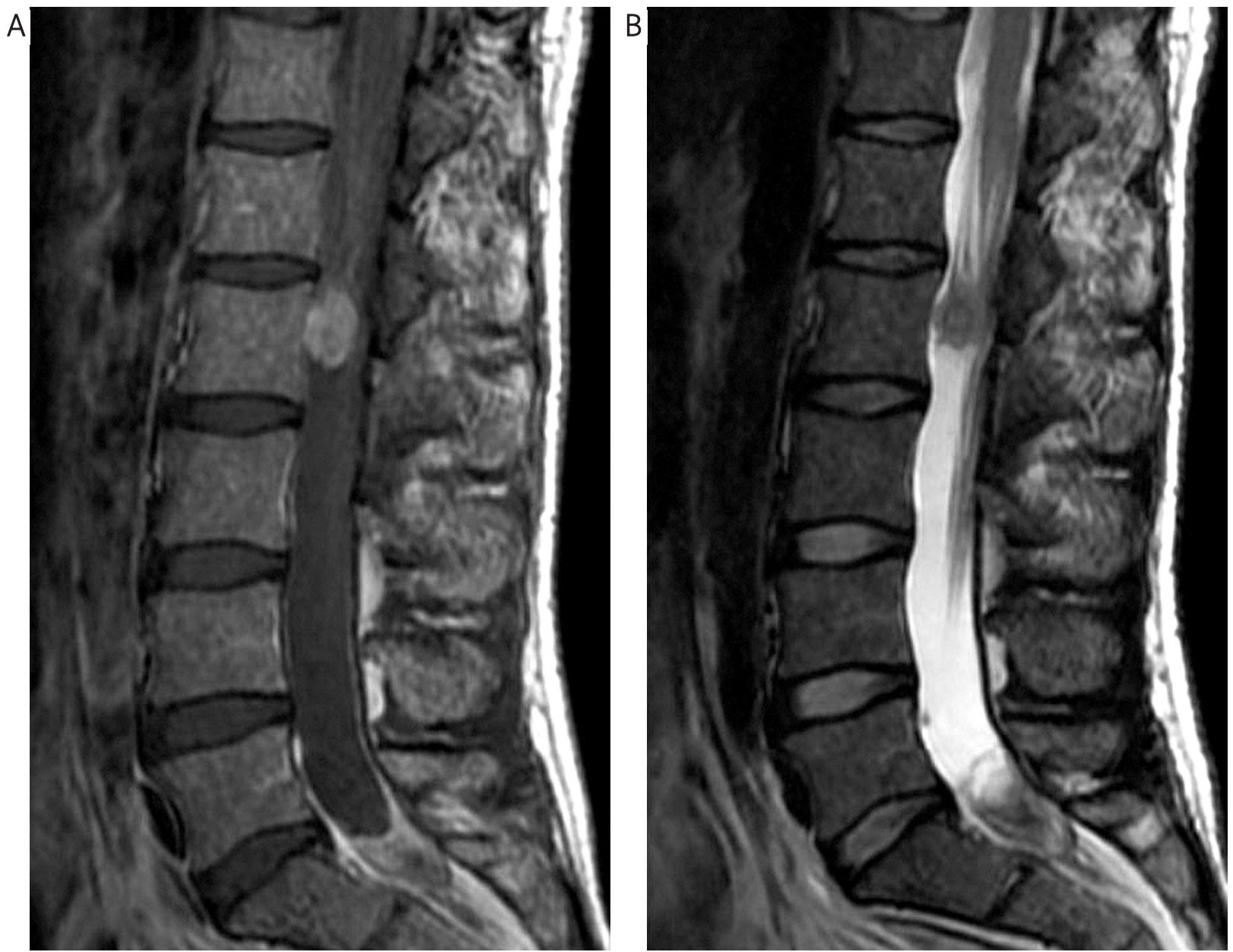

Fig. 1. A sagittal $\mathrm{T} 1$ weighted spin echo image with contrast enhancement $(\mathbf{A})$ and $\mathrm{T} 2$ weighted spin echo image (B), both showing an oval intradural tumourous formation in the conus medullaris region just behind the vertebra L2. No disc pathology is seen. 


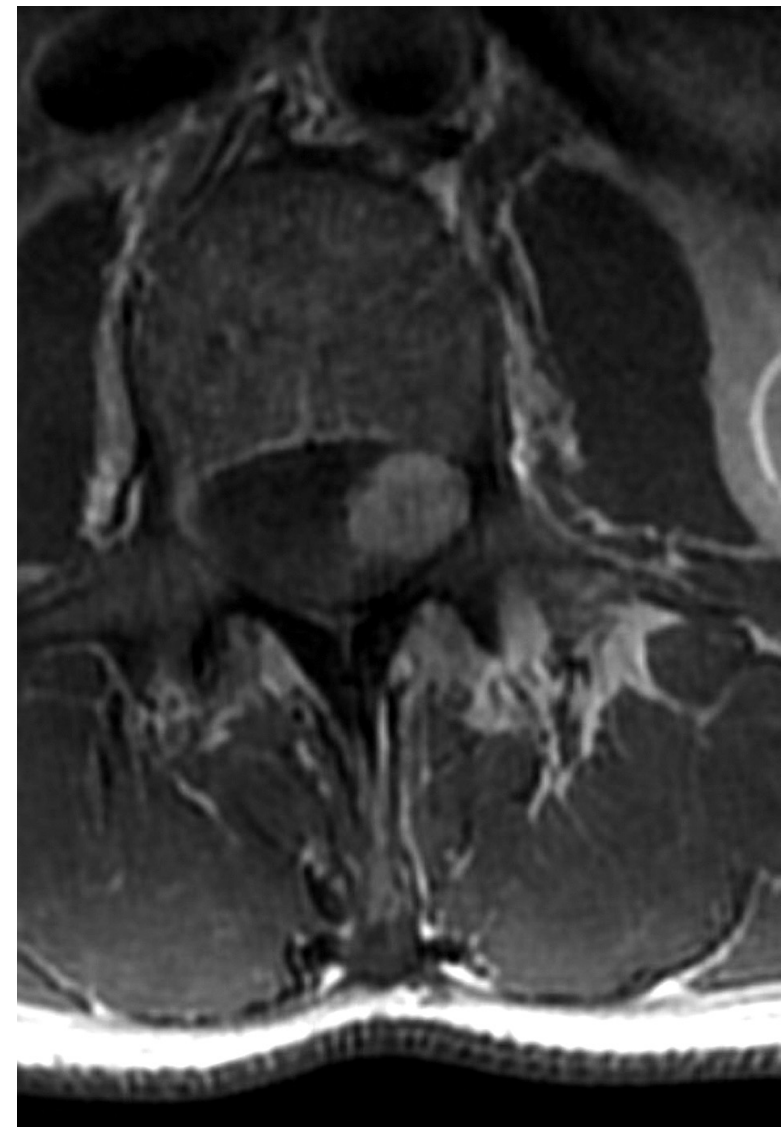

Fig. 2. An axial T1 weighted spin echo image with contrast enhancement. An intradural mass of $2 \mathrm{~cm}$ in diameter in the conus medullaris at the level L2, slightly on the left side of the spinal cord, the compression of the cord is evident.

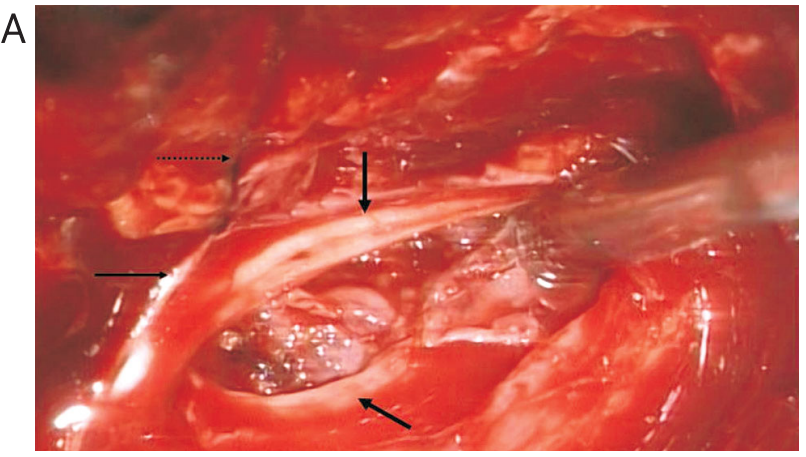

B

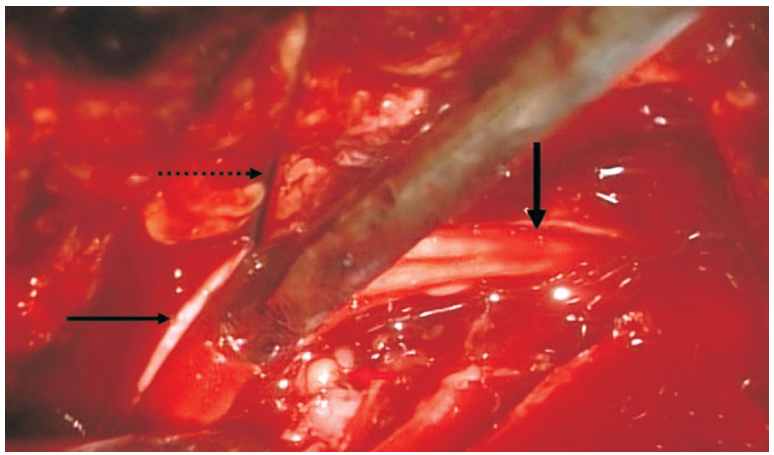

Fig. 3. An intraoperative view of the fibrous capsule of the abscess in the conus medullaris region, strongly adherent to and intermingled with spinal nerve roots. The suction catheter is used to expose the capsule and gently shift the roots aside (thick arrows). The dural flaps (thin arrows) are attached to holding sutures (dotted arrow) (A). During the dissection of the capsule, a few millilitres of pus were drained from the formation (arrow) (B). tumourous mass of grey colour was found on the left side of the conus medullaris, strongly adherent to the spinal nerves forming the cauda equina. During the dissection, a few millilitres of pus were drained from the formation (Fig. 3). At this point, it became evident that the tumourous mass was an abscess. Swabs were taken and the thick fibrous capsule was carefully dissected from the nerves. Although strongly adherent, it was removed completely and no adherent nerves running across were damaged. The wound was irrigated and closed in layers. The frozen section showed granulation tissue with signs of acute and chronic inflammation, as was later confirmed during the definitive histological examination (Fig. 4). No tuberculosis bacilli were found.

Postoperatively, the patient received empirical antibiotic treatment with vancomycin and cefotaxime.
The swabs were sterile. No signs of virulence were seen in the recovery period and the strength of the left foot gradually improved (Fig. 5). The rest of the hospitalization was uneventful.

\section{Discussion}

Back pain is a complex and frequent complaint, however, numerous life-threatening or disabling conditions may present with signs and symptoms similar to those of more benign disease processes [2]. Spinal intradural abscess, as experienced by our patient, is a seldom described clinical condition [4,17]. Even rarely, spinal intradural abscess may be the cause of sciatica, thus being easily mistaken for another form of pathology [1,12]. In contrast to more frequent spinal epidural abscesses, where lumbar vertebrae are 

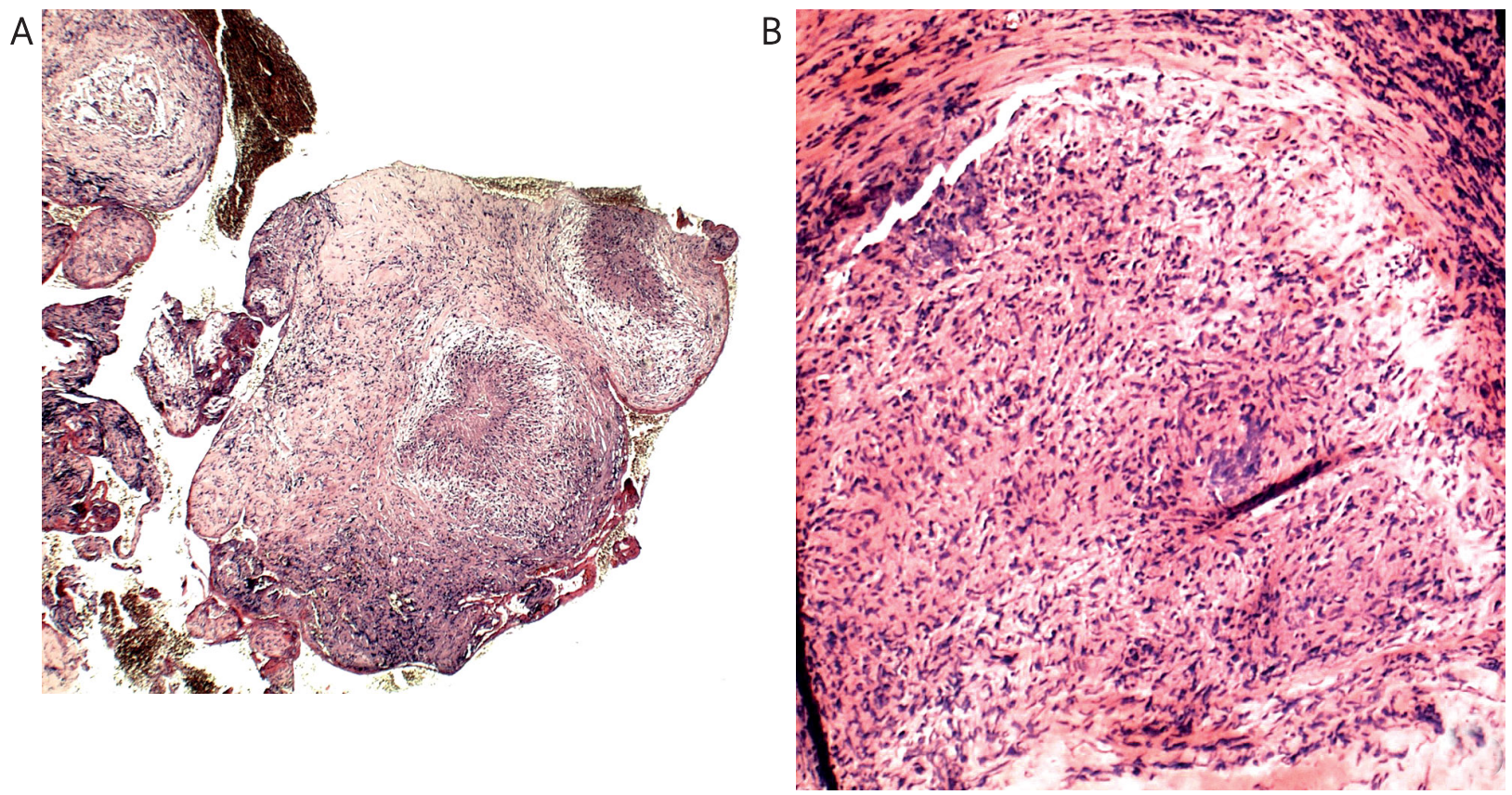

Fig. 4. A microphotograph of the excised specimen. Two granulomas surrounded by chronically inflamed granulation tissue can be seen (magnification: 10x) (A). A granuloma consisting of central necrosis and surrounded by palisading histiocytes (magnification: 100x) (B).

the most common region of involvement, spinal intradural abscess may be formed anywhere along the spinal cord, most commonly involving the thoracic region $[3,10,12]$. Thus, a high level of suspicion must be present when managing a patient with back pain in order to avoid confusion with more common spinal problems, for example a herniated disc [2]. In our patient, the history and clinical examination with a decreased strength of dorsal flexion of the foot and toe clearly pointed to this more trivial and common form of spinal pathology.

It is vital to recognise clinical features of the spinal intradural abscess instantly to arrange appropriate diagnostic work-up and treatment, especially when the patient complains of spinal pain or a neurologic deficit in combination with fever or an elevated erythrocyte sedimentation rate and pathological laboratory findings $[1,16,17]$. Neuroimaging is important in setting the final diagnosis [8]. As our case showed however, neuroimaging is not ultimate either. Radiologically, the intradural mass was suspected to be a neurofibroma or some other form of a tumour. Intraoperatively, an abscess was discovered. It is possible that the signal on MRI was different due a thick capsule of granulation tissue embracing the abscess and a re- latively small amount of liquid material (i.e. pus) in the collection, radiologically confusing it with a tumour.

Appropriate treatment of intradural abscess should be initiated promptly as morbidity and mortality still remain significant. Intradural abscess, similarly to epidural abscess, has a poor prognosis due to accumulation of purulent material, compressing the spinal cord and compromising its vascular supply, although reports of a good treatment outcome have been described $[4,6,17,19]$. The most effective treatment of spinal intradural abscess is an immediate surgical evacuation followed by a long-term antimicrobial therapy, which may be adjusted according to the results of antibiogram $[7,15,17]$. From our experience, we agree that a prompt surgical treatment should be performed particularly in cases with severe neurologic deficits. Urgent surgical decompression and debridement followed by a longterm antimicrobial therapy must have priority over medicamentous treatment for the best treatment outcome $[15,17]$. The therapeutic strategy consists of a surgical drainage and systemic antibiotic therapy for four to six weeks [15]. However, the literature is not consistent when the patients are asymptomatic. It has been described that in the absence of a neurologic impairment, medical management is an alternative to 

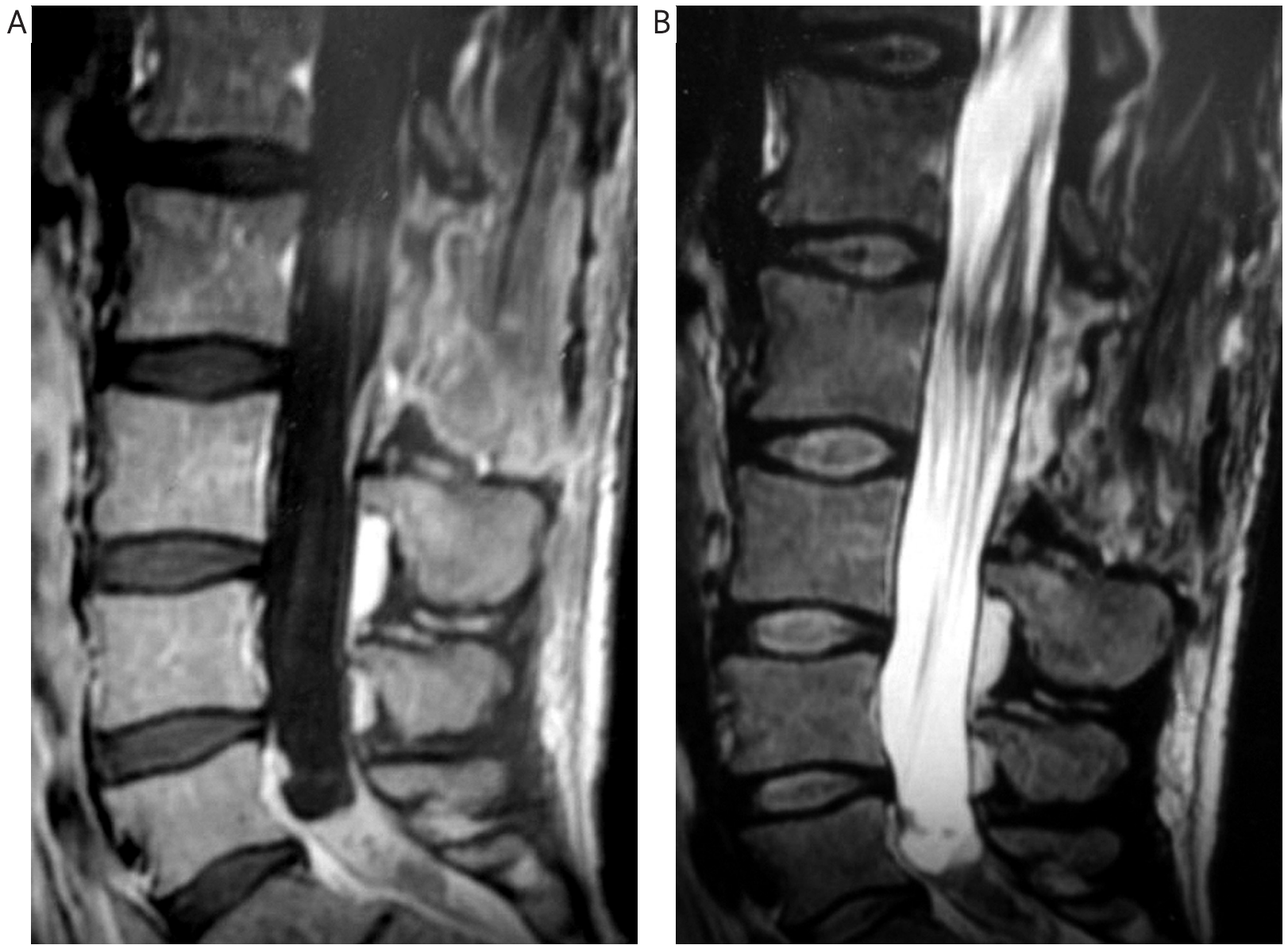

Fig. 5. A sagittal T1 weighted spin echo image with contrast enhancement (A) and T2 weighted spin echo image (B) after surgical evacuation of the abscess. Postoperative oedema and inflammation in the conus medullaris region can be seen as well as removed lamina L2 after laminectomy.

surgery when the risk of neurologic complications is low based on the location and morpholog of the abscess, immune status of the patient and virulence of the organism. In such carefully selected patients, surgical intervention may be avoided and nonoperative management can be cautiously considered [8]. On the other hand, there is a significant risk of sudden neurologic deterioration in cases managed conservatively [4]. Due to the neurologic deficit, we have decided for surgery first and then instantaneous empirical antibiotic treatment followed. As the swabs were negative and the patient was recovering well with no signs and symptoms of local or systemic inflammation, the antibiotics were not changed during the treatment course and were discontinued after four weeks with no late sequelae.

Data describing the aetiology of intradural abscess are rare. It is assumed that its formation may be found in haematogenous seeding from a distant focus and after the spinal surgery or medical procedure in a nearby region, although this is more typical of the epidural abscess $[9,11,13,15-18]$. It is possible that the intradural abscess of our patient started to form after the lumbar puncture in the diagnostic course of chronic headache, which was performed six months before the clinical manifestation of the abscess. Slowly, inflammation started to develop in the intradural space near the puncture site, although a bit high according to the usual location of the lumbar punctures at the level L3 or L4. Following a few months' course, the local infection progressed slowly, involving the neural structures. The absence of systemic clinical signs and elevated inflammatory parameters at admission may be explained by a thick capsule surrounding the abscess, which confined the inflammation to one place and prevented it from spreading widely. 
It is interesting how the history and physical examination in combination with neuroimaging may sometimes act as a diagnostic pitfall, the true pathology becoming evident only after the surgical intervention. Despite the advent of modern medicine, it is not always possible to predict the exact diagnosis, but it is the modern medicine on the other hand that helps patients to recover in diseases otherwise not treatable.

\section{Conclusions}

Intradural abscess is an infrequent and dangerous form of pathology. Thus, a high level of suspicion must be present when treating a patient with back pain, neurologic symptoms and signs of infection. For the best treatment outcome, immediate spinal decompression and evacuation of the abscess is required in conjunction with an appropriate antimicrobial therapy, principally in cases with neurologic deficits.

\section{References}

1. Achouri M, Hilmani S, Sami A, Ouboukhlik A, el Kamar A, el Azhari A, Boucetta M. Intradural extramedullary tuberculous abscess. Apropos of a case. Neurochirurgie 1996; 42: 306-308.

2. Broder J, Snarski JT. Back pain in the elderly. Clin Geriatr Med 2007; 23: 271-289.

3. Daglioglu E, Bayazit N, Okay O, Dalgic A, Hatipoglu HG, Ergungor F. Lumbar epidural abscess caused by brucella species: report of two cases. Neurocirugia (Astur) 2009; 20: 159-162.

4. DeSanto J, Ross JS. Spine infection/inflammation. Radiol Clin North Am 2011; 49: 105-127.

5. Kanayama M, Togawa D, Takahashi C, Terai T, Hashimoto T. Crosssectional magnetic resonance imaging study of lumbar disc degeneration in 200 healthy individuals. J Neurosurg Spine 2009; 4: 501-507.

6. Kim MS, Kim KJ, Chung CK, Kim HJ. Intradural extramedullary tuberculoma of the spinal cord: a case report. J Korean Med Sci 2000; 15: 368-370.

7. Lange M, Tiecks F, Schielke E, Yousry T, Haberl R, Oeckler R. Diagnosis and results of different treatment regimens in patients with spinal abscesses. Acta Neurochir (Wien) 1993; 125: 105-114.

8. Manelfe C. Imaging of the spine and spinal cord. Curr Opin Radiol 1991; 3: 5-15.

9. Maniadakis N, Gray A. The economic burden of back pain in the UK. Pain 2000; 84: 95-103.

10. Muthukumar N, Sureshkumar V, Ramesh VG. En plaque intradural extramedullary spinal tuberculoma and concurrent intracranial tuberculomas: paradoxical response to antituberculous therapy. Case report. J Neurosurg Spine 2007; 6: 169-173.

11. Nadkarni T, Shah A, Kansal R, Goel A. An intradural-extramedullary gas-forming spinal abscess in a patient with diabetes mellitus. J Clin Neurosci 2010; 17: 263-265.

12. Ozek E, Iplkcioglu AC, Erdal M. Intradural extramedullary tuberculoma mimicking en plaque meningioma. Neurol India 2009; 57 211-212.
13. Saigal G, Donovan Post MJ, Kozic D. Thoracic intradural Aspergillus abscess formation following epidural steroid injection. AJNR Am J Neuroradiol 2004; 25: 642-644.

14. Sillevis Smitt $P$, Tsafka A, van den Bent $M$, de Bruin $H$, Hendriks W, Vecht C, Teng-van de Zande F. Spinal epidural abscess complicating chronic epidural analgesia in 11 cancer patients: clinical findings and magnetic resonance imaging. J Neurol 1999; 246: 815-820.

15. Stabler A., Reiser M.F. Imaging of spinal infection. Radiologic Clinics of North America 2001; 39: 115-135.

16. Tanriverdi T, Kizilkiliç O, Hanci M, Kaynar MY, Unalan H, Oz B. Atypical intradural spinal tuberculosis: report of three cases. Spinal Cord 2003; 41: 403-409.

17. Thomé C, Krauss JK, Zevgaridis D, Schmiedek P. Pyogenic abscess of the filum terminale. Case report. J Neurosurg 2001; 95: 100-104.

18. Ugarriza LF, Porras LF, Lorenzana LM, Rodríguez-Sánchez JA, García-Yagüe LM, Cabezudo JM. Brucellar spinal epidural abscesses. Analysis of eleven cases. Br J Neurosurg 2005; 19: 235-240.

19. von Albert HH. Lumbalgia, lumboischialgia. 2: Instrumental diagnosis, differential diagnosis. Fortschr Med 1990; 108: 363-364. 